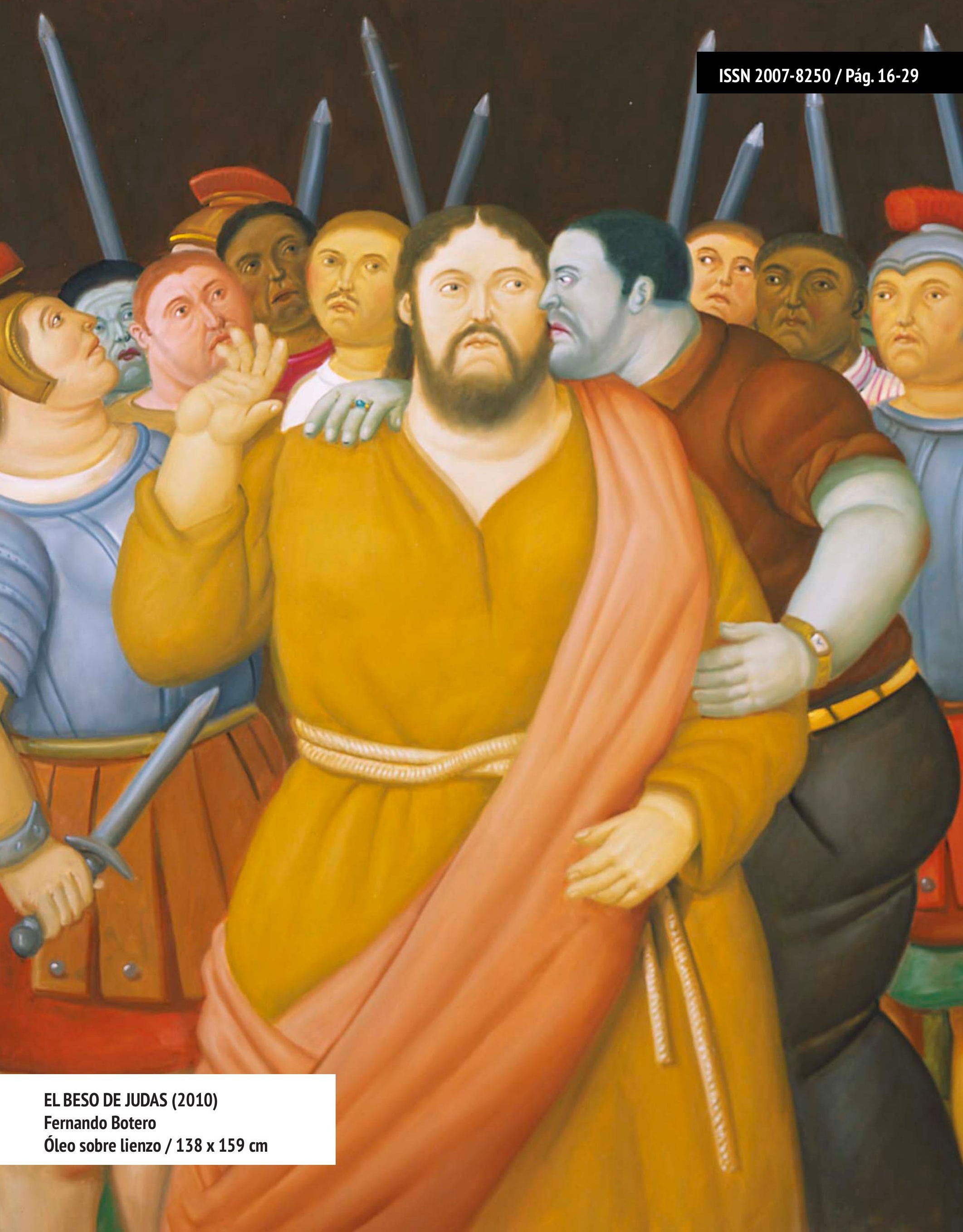




\section{EL PROYECTO RECREA Y SU PROPUESTA METODOLÓGICA PARA LA TRANSFORMACIÓN DE LOS PROCESOS FORMATIVOS EN LA UNIVERSIDAD}

○

THE PROPOSAL METHODOLOGICAL OF RECREA PROJECT FOR THE TRANSFORMATION OF TRAINING PROCESSES IN THE UNIVERSITY

\section{EMMA ISABEL RODRÍGUEZ RUVALCABA FELIPE ABUNDIS DE LEÓN}

RESUMEN

Desde el año 2017, por iniciativa de la Secretaría de Educación de México, se creó el Proyecto RECREA (Red de Comunidades para la Renovación de la Enseñanza Aprendizaje en Educación Superior) el cual tiene como finalidad generar un cambio y transformación en las tareas de enseñanza que se llevan a cabo en educación superior, particularmente en las Escuelas Normales de México, favoreciéndose de los aportes que en el campo de la investigación educativa pueda brindarles las universidades públicas. La necesidad de proponer este modelo de intervención en el aula radica en la importancia de formar a los futuros formadores en nuevas estrategias, paradigmas, visiones y tendencias sobre las cuales gravita la educación superior en el contexto actual, no olvidando que los agentes educativos del mañana requieren tener una sólida formación para responder de manera cabal a los retos que imponen las sociedades del siglo XXI, todo esto con el fin de innovar la enseñanza en la universidad.

PALABRAS CLAVE: Educación - Innovación - Enseñanza - Aprendizaje - Académicos.
ABSTRACT

Since 2017, at the initiative of the Ministry of Education of Mexico, the RECREA Project (Network of communities for the renewal of teaching-learning in Higher Education) was created, which aims to generate a change and transformation in teaching tasks that are carried out in higher education, particularly in the Normal schools of Mexico, benefiting from the contributions that public universities can provide in the field of educational research. The need to propose this intervention model in the classroom lies in the importance of training future trainers in new strategies, paradigms, visions and trends on which higher education gravitates in the current context, not forgetting that the educational agents of tomorrow They require having a solid training to fully respond to the challenges posed by 21st century societies, all with the aim of innovating teaching at the university.

KEYWORDS: Education - Innovation - Teaching - Learning - Academic. 
D esde el año 2017, por iniciativa de la Secretaría de Educación de México, se creó el Proyecto RECREA (Red de Comunidades para la Renovación de la Enseñanza Aprendizaje en Educación Superior) el cual tiene como finalidad generar un cambio y transformación en las tareas de enseñanza que se llevan a cabo en educación superior, particularmente en las Escuelas Normales de México, favoreciéndose de los aportes que en el campo de la investigación educativa pueda brindarles las universidades públicas.

La necesidad de proponer este modelo de intervención en el aula radica en la importancia de formar a los futuros formadores en nuevas estrategias, paradigmas, visiones y tendencias sobre las cuales gravita la educación superior en el contexto actual, no olvidando que los agentes educativos del mañana requieren tener una sólida formación para responder de manera cabal a los retos que imponen las sociedades del siglo XXI.

Si bien es cierto que la tarea docente exige cada vez más una mejor comprensión de los cambios y transformaciones que se están dando en todos los ámbitos de la vida social, para así poder planear, diseñar e instrumentar una serie de acciones pedagógicas que respondan a los nuevos escenarios donde se insertan nuestros estudiantes, también es cierto que esto nos demanda una mayor actualización como agentes educativos, una formación continua en nuestro campo de especialización, la necesidad de migrar a una cultura digital que se impone con gran fuerza y al entendimiento de los nuevos roles y funciones que nos son demandados como académicos.

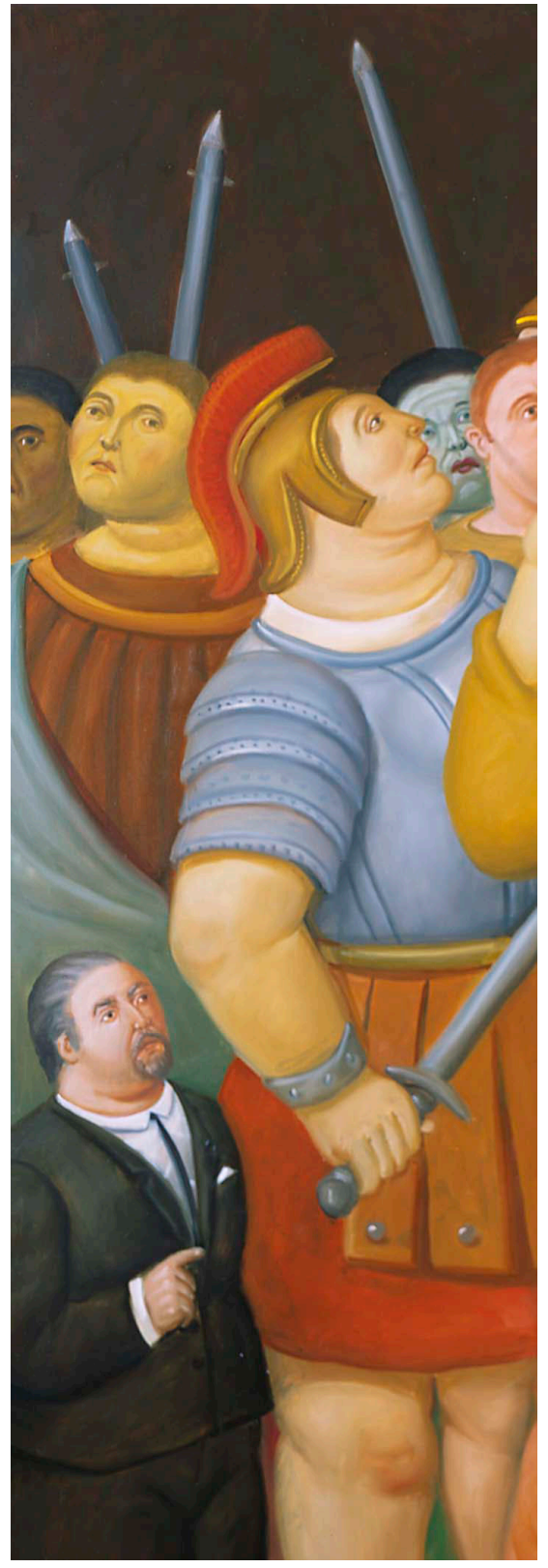

La tarea de la enseñanza es compleja por los múltiples factores que intervienen en la misma y las orientaciones que le imprimen aquellos que están a cargo de la conducción de los procesos formativos; de allí que cada práctica sea única, diferenciada y cargada de diversos significados, dependiendo de quién, dónde y bajo qué condiciones la lleve a cabo. Los diversos factores que distinguen una práctica son dignos de análisis y reflexión para poder conocer y avizorar los resultados que se pueden llegar a obtener y ver la calidad del proceso según la forma en que se opera. De allí que la reflexión sobre la tarea de la enseñanza y el conocimiento de todos los elementos constitutivos de la misma sean hasta hoy objeto de interés y de estudios por los profesionales de la educación y la pedagogía, para revitalizar esta actividad, adecuarla a las necesidades actuales e innovar los procesos de aprendizaje resultados de la misma.

Con base en lo anterior, la creación del proyecto RECREA se circunscribe en la necesidad de proponer nuevas formas de planear la enseñanza, operar los procesos diseñados con antelación, evaluar la pertinencia del trabajo

La necesidad de proponer este modelo de intervención en el aula radica en la importancia de formar a los futuros formadores en nuevas estrategias, requieren tener una sólida formación para responder de manera cabal a los retos que imponen las sociedades del siglo XXI 


\section{RECREA constituye un camino para transitar}

\section{a una mejor clarificación de las tareas}

\section{del enseñante para organizar los procesos}

\section{de enseñanza e incorporar en la planeación}

didáctica saberes, procedimientos y formas

de actuación que inciden en una formación

integral, de calidad y con visión de futuro

realizado y volver al punto de partida del diseño. Para ello y con el fin de dar concreción a este proyecto, se determinaron desde su inicio 4 grandes ejes que de manera articulada dan cohesión a los trabajos de formación y capacitación de quienes operan esta metodología, proponiendo a la vez como una alternativa que permitirá, para aquellos agentes educativos que así lo requieran, una forma nueva de planear el proceso educativo, ajustarlo a los tiempos modernos y contribuir a procesos de aprendizaje más significativos, acordes a las necesidades reales del campo laboral y las nuevas orientaciones pedagógicas.

Conviene señalar que a partir de finales del siglo XIX y sobre todo en el siglo XX, la humanidad sufrió cambios vertiginosos en todos los campos y latitudes geográficas; la renovación y el cambio se han constituido en toda una cultura de la cual se habla en todas las esferas de la actividad humana y a la cual están abocados todos los sistemas, instituciones, gremios, etc., con pena de quedar en el aislamiento 0 en la obsolescencia absoluta. En este sentido, la escuela, como ente social y la importante función que desempe-

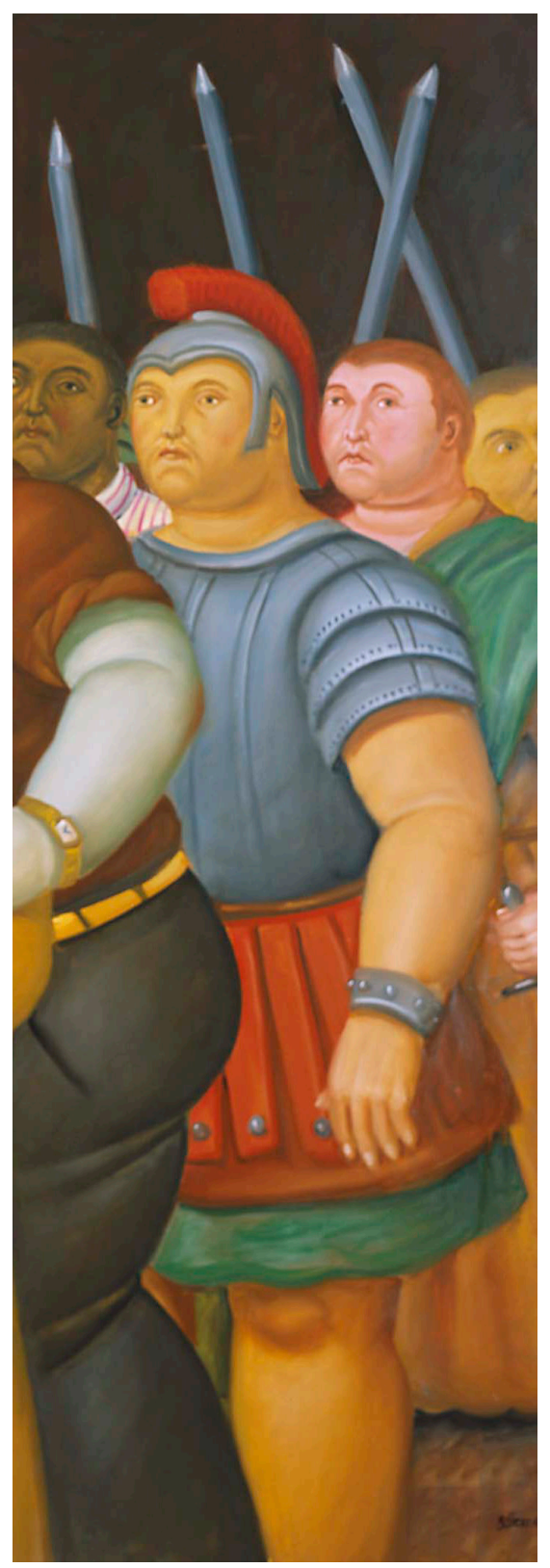

ña, está convocada a hacer suya esta visión de transformación y renovación de los que es y lo que hace, reconsiderando sus fines, orientaciones y adaptándose a nuevas realidades que demandan de ella un continuo flujo de innovaciones, modificaciones y renovaciones desde su interior.

En virtud de lo anterior, RECREA constituye un camino para transitar a una mejor clarificación de las tareas del enseñante, una mejor pericia para organizar de mejor forma los procesos de enseñanza e incorporar en la planeación didáctica saberes, procedimientos y formas de actuación que inciden en una formación integral, de calidad y con visión de futuro en los estudiantes que se forman en nuestras aulas. A lo largo del desarrollo de este proyecto, todos los académicos que hemos tenido la oportunidad de ser capacitados y formados en esta propuesta de innovación pedagógica, hemos podido advertir los nuevos significados que nuestra práctica ha tenido, la necesaria búsqueda de nuevas fuentes de información para ofrecer a nuestros estudiantes, la incorporación de la tecnología para hacer más eficientes nuestros trabajos, pero constituirnos a la vez en investigadores de nuestra propia práctica, generando nuevas reflexiones y saberes, estando en la capacidad y las condiciones de mejorar nuestra tarea como docentes.

Si bien es cierto que la observación y análisis de cualquier práctica docente revela no sólo la formación del maestro, sino también sus orientaciones ideológicas, capacidad para transmitir informaciones y generar conocimientos, sus destrezas para organizar y ofrecer saberes de manera pertinente, también es cierto que las diferencias 
que podemos encontrar en cada ejercicio de enseñanza revela la capacidad del profesor por hacer de ésta un arte y de contribuir con la misma a la formación de seres humanos capaces de aprender, desarrollar habilidades e ir consolidando y encarnando en ellos una serie de valores que les permitan no sólo un desarrollo humano pertinente, sino también la consolidación de una personalidad equilibrada para un ejercicio profesional satisfactorio.

Algunos profesionales de la educación afirman que en una sociedad donde el conocimiento y las habilidades se van tornando en obsoletas e incluso tienden a convertirse en rutina, todos los miembros de la sociedad pasan a formar parte del proceso creativo, tal vez no generando ideas, pero sí aportando e incorporando en sus vidas nuevas ideas, conceptos y habilidades (Goldberg, 2018) y esto atañe a todo los ámbitos sociales, incluyendo el educativo. Es así como los sistemas educativos deberán cambiar para poder estar en condiciones de preparar a las personas para la vida, desde una visión

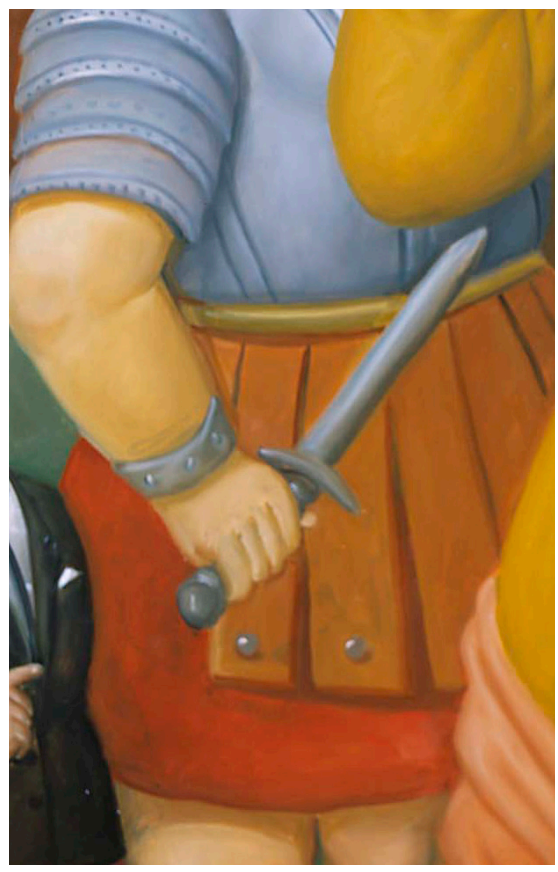

en 2 sentidos: desde la sociedad en las que se desarrollan pero también en las sociedades donde vivirán en un futuro próximo.

Si bien es cierto que para poder operar procesos educativos se necesita tener un conocimiento profundo del contexto donde vivimos, los recursos de los que se dispone para la tarea educativa y el tipo de agente educativo que se requiere para operar los procesos formativos, también es necesario tener claridad hacia donde se dirigen nuestros esfuerzos como escuelas y como educadores, teniendo claridad sobre la sociedad que deseamos construir, el tipo de hombre y de mujer que se encuentran en nuestros idearios pedagógicos y el mundo que deseamos transformar con nuestra tarea como profesores. Sin olvidar los legados que hemos recibido de generaciones anteriores, necesitamos hacer una relectura del contexto, renovar nuestra formación con nuevos saberes, abrirnos a nuevas realidades y escenarios y cultivar la cultura de la innovación educativa en nuestro trabajo diario.

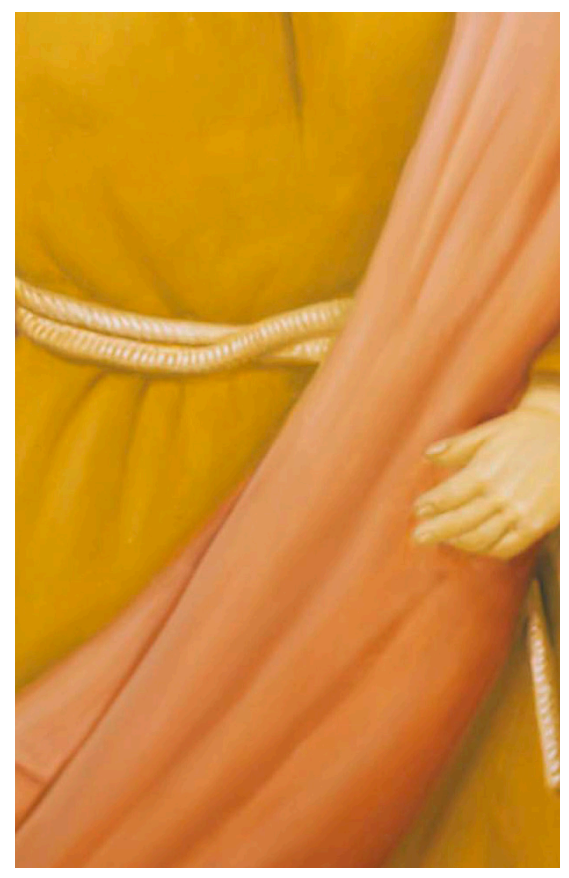

En virtud de lo anterior, es nuestro interés dar a conocer cómo desde la propuesta metodológica del proyecto RECREA, incorporando a las tareas de enseñanza los ejes que plantea, se pueden ir transformando gradualmente las formas como enseñamos en todos los niveles educativos y cómo desde este nuevo enfoque podemos ir transitando los académicos y académicas hacia nuevas formas de hacer educación y responder con ello de manera más oportuna a los retos que la sociedad contemporánea nos demanda.

Esta es una tarea emergente y todas las instituciones educativas necesitan orientar sus esfuerzos a cambiar gradualmente y transformarse en todo lo que les conforma.

\section{CONTEXTUALIZACIÓN}

Conviene señalar que en las instituciones educativas de México tienen muchas reminiscencias de la escuela tradicional y particularmente las prácticas de enseñanza poseen un tinte ritualista donde da la impresión de

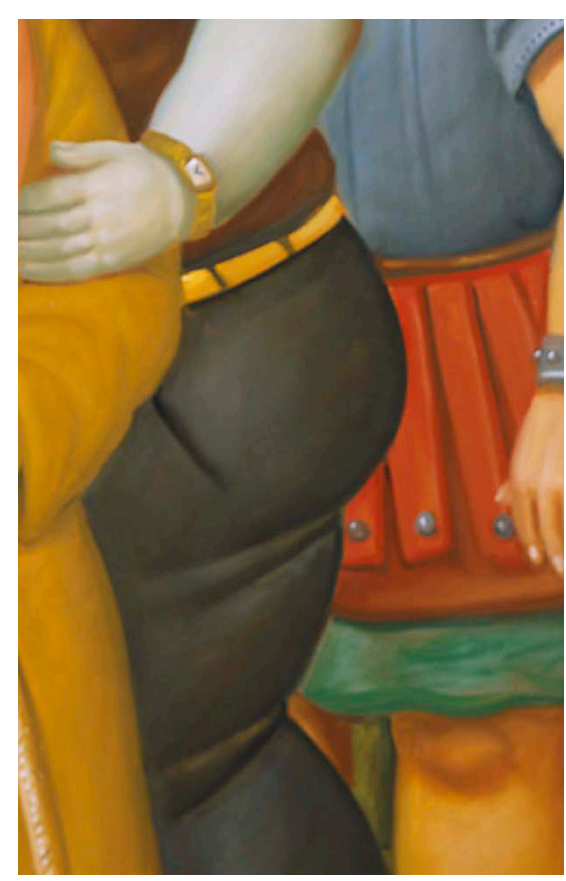




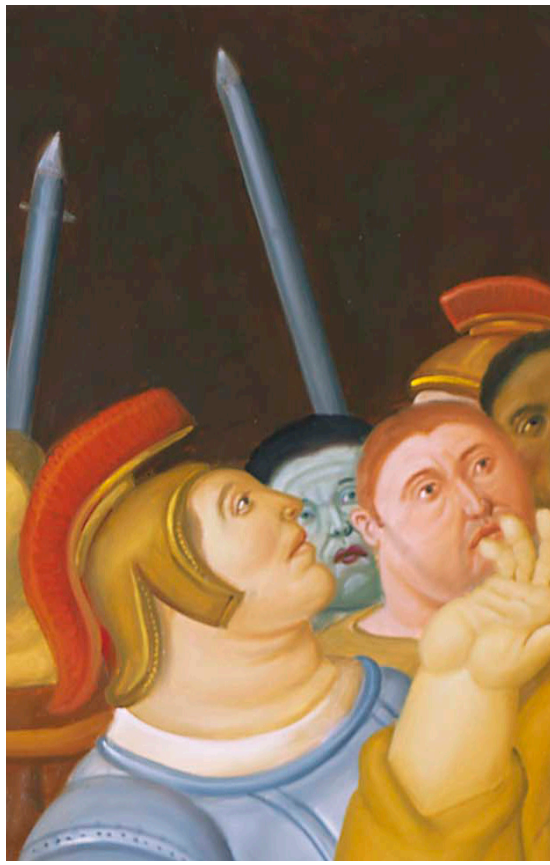

que lo que ocurre en esta tarea está predeterminado y en algunos casos fragmentado. En otros momentos, pudiera pensarse que se hace enseñanza sin tener un propósito determinado, sino sólo con la consigna de llevar a cabo una tarea para la cual ha sido formado 0 , en algunos casos, operada desde el empirismo más elemental. Es así como la transformación de los procesos de enseñanza, la estructuración de ejes orientadores para llevar a cabo la misma, el buscar en todo momento el desarrollo integral del aprendiente, sea una tarea insoslayable y a la cual hay que abocarnos.

Con el fin de contribuir a esta cultura del cambio en la enseñanza, el proyecto RECREA nos ha propuesto 3 grandes ejes para planear la enseñanza y lograr aprendizajes más significativos en nuestros estudiantes. Estos ejes están en consonancia con las tendencias actuales de la educación y están a la vez considerados en los modelos educativos de vanguardia, por lo cual fueron propuestos como caminos ideales que nos permitirán arribar metas edu-

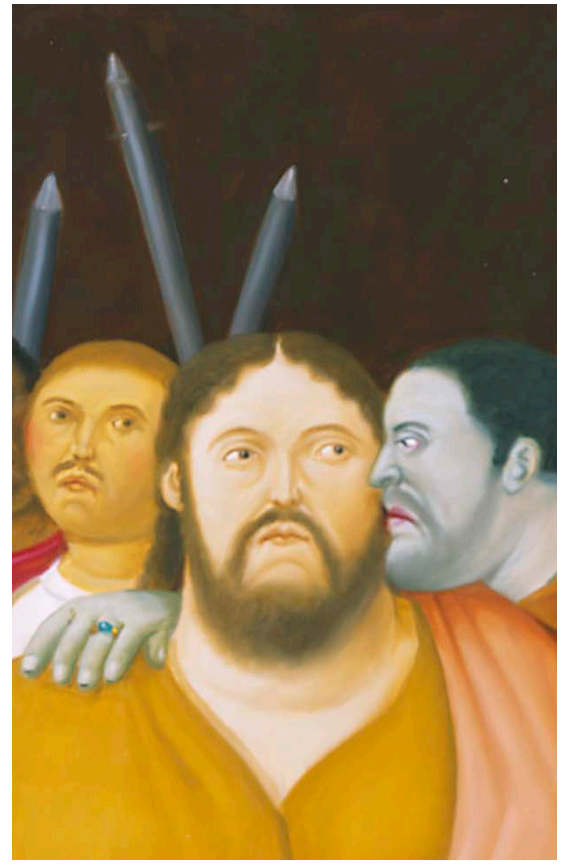

cativas de excelencia. A continuación analizaremos cada uno de estos ejes y la manera de integrarnos a la planeación didáctica.

\section{EJE 1. PENSAMIENTO COMPLEJO}

La teoría de la complejidad propuesta por Edgar Morín vino a resignificar de manera sustancial la forma de entender el saber en el siglo XX. Algunos autores como Torres (2013) afirman que su uso común era relacionado con lo complicado, enmarañado y difícil de entender, pero que ahora es posible entenderlo desde una perspectiva diferente para poder designar al ser humano, la naturaleza y las relaciones que nosotros como sujetos tenemos con ella.

Lo complejo, en palabras de Morín (2004), designa hoy una comprensión del mundo como entidad donde todo se encuentra entrelazado, como un tejido compuesto de finos hilos. De igual manera, el autor señala, siguiendo su idea, que el pensamiento complejo es ante todo un pensamiento que relaciona y que entrelaza todos los

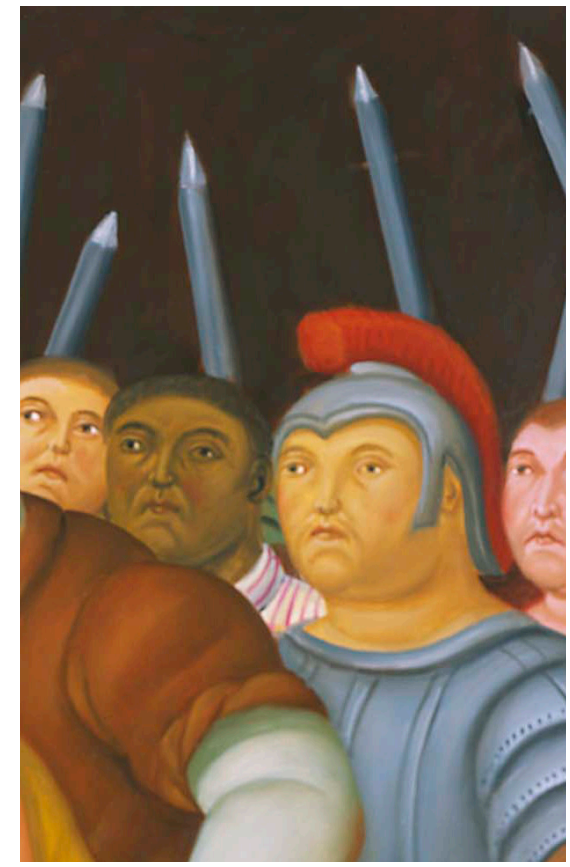

elementos que coexisten en una realidad determinada. Es el significado más cercano del término complexus (lo que está tejido en conjunto). Esto nos lleva a inferir que en oposición al modo de pensar tradicional, que divide el campo de los conocimientos en disciplinas atrincheradas y clasificadas, el pensamiento complejo es un modo de religación (unión). Ésta contra el aislamiento de los objetos de conocimiento, poniéndolos en su contexto y, de ser posible, en la globalidad a la que pertenecen.

Es así como el pensamiento complejo de Morín es reconocido como un pensamiento que relaciona y complementa, que no excluye ni aleja, sino que conexiona, articula y aglutina, donde su objeto y sujeto de estudio es el todo, a través de sus efectos, defectos, dinamismo y estática, reconociendo la interrelación del todo con sus partes y viceversa, dentro de un entramado no siempre fácil de entender. De esta manera, podemos afirmar sin temor a equivocarnos que el estudio del pensamiento complejo hoy día ha impac- 
tado en el ámbito más directo de las interacciones entre los seres humanos, específicamente en campos como la educación, la interpretación de la sociedad, la política y la comprensión del momento actual que vive la humanidad.

El problema de la complejidad, de acuerdo a Torres (2013), ha pasado a ser el problema de la vida y el cómo vivir; el problema de la construcción del futuro y la búsqueda de soluciones a los problemas contemporáneos que aquejan a la humanidad, y cita a Morín cuando éste afirma que: cuando se habla de la complejidad se trata de enfrentar la dificultad del pensar y del vivir, cuando habla de esto en su libro titulado El Método.

Nos parece también importante mencionar que la teoría del pensamiento complejo asume la Heterogeneidad, la Interacción y el Azar, así como la totalidad sistémica, la cual se funda en 3 principios: Principio Dialógico, Principio de Recursividad y Principio Hologramático, mismos que se explican a continuación:

1. Principio Dialógico: No asume la superación de los contrarios, sino que los dos términos coexisten sin dejar de ser antagónicos; este principio valora el grado máximo de conexión como condición del sistema.

2. Principio de Recursividad: El efecto se vuelve causa, la causa se vuelve efecto; los productos son productores, el individuo hace cultura y la cultura hace a los individuos.

3. Principio Hologramático: Busca superar el principio del holismo y del reduccionismo. El holismo no ve más que el todo; el reduccio-

\section{El problema de la complejidad ha pasado}

a ser el problema de la vida y el cómo vivir; el problema de la construcción del futuro y la búsqueda de soluciones a los problemas contemporáneos que aquejan a la humanidad

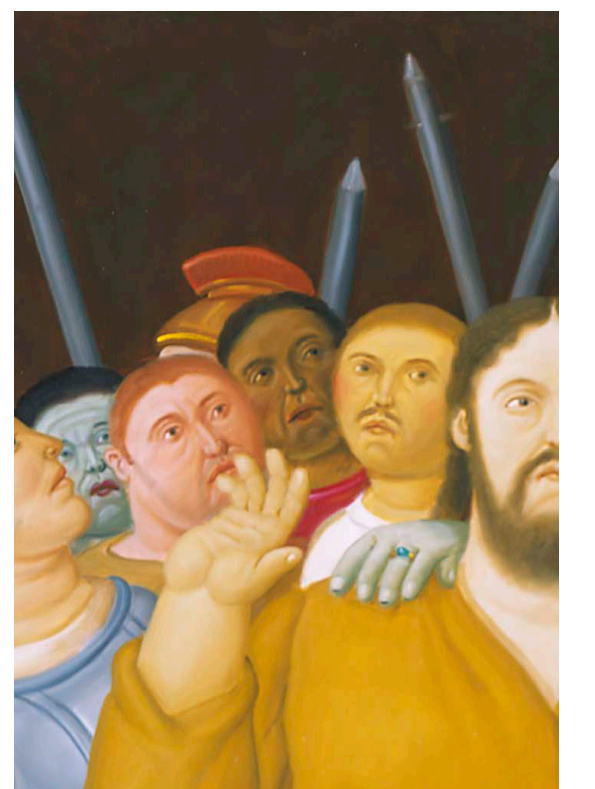

nismo no ve más que las partes. El Principio Hologramático ve las partes en el todo y el todo en las partes.

De esta manera, Morín, al postular estos principios, aduce 2 conceptos que están interrelacionados: el Paradigma y el Sujeto. El primero lo define como la estructura mental y cultural bajo la cual se mira la realidad, y el segundo lo conceptúa como toda realidad viviente que está caracterizada por la autonomía, la individualidad y su capacidad para procesar la información y convertirla en conocimiento. Para este autor, el sujeto es el de mayor complejidad, ya que sostiene que no se puede asumir esta noción de sujeto desde un paradigma simplista.

Es necesario el pensamiento complejo, aquel que es capaz de unir conceptos que se rechazan entre sí y que son desglosados y catalogados en compartimentos cerrados por el pensamiento no complejo. No se trata pues de rechazar lo simple, se trata de verlo articulado con otros elementos y esto requiere la habilidad de separar y enlazar al mismo tiempo. Se trata, en síntesis, de comprender un pensamiento 
que separa y que reduce junto a otro pensamiento que distingue y enlaza.

\section{DESCRIPCIÓN DE LA EXPERIENCIA}

Dentro del proyecto RECREA, este primer eje propuesto en el diseño instruccional nos lleva plantearnos la manera en cómo vemos los saberes, la forma en que los articulamos y si hacemos fragmentaciones del mismo 0 tenemos la capacidad de ver el conocimiento como un todo que se puede desarticular para un mejor análisis, para luego volver a integrarlo en el todo. No se trata de fragmentar un objeto, desvinculándolo de otros, sino educando desde una visión de unidad, complementariedad e integración del todo a través de sus partes. Incluir en nuestras planeaciones el pensamiento complejo requiere de una seria reflexión sobre nuestros propios procesos de aprendizaje, la forma cómo fuimos aprendiendo, la manera en que nuestros formadores nos fueron acercando a los objetos de estudio y si sólo nos redujeron a un campo de conocimiento, desvinculado de los otros, 0 si nos ayudaron a ver en él el todo que está constituido en varias partes. Es así como la utilización del pensamiento complejo puede ayudarnos a

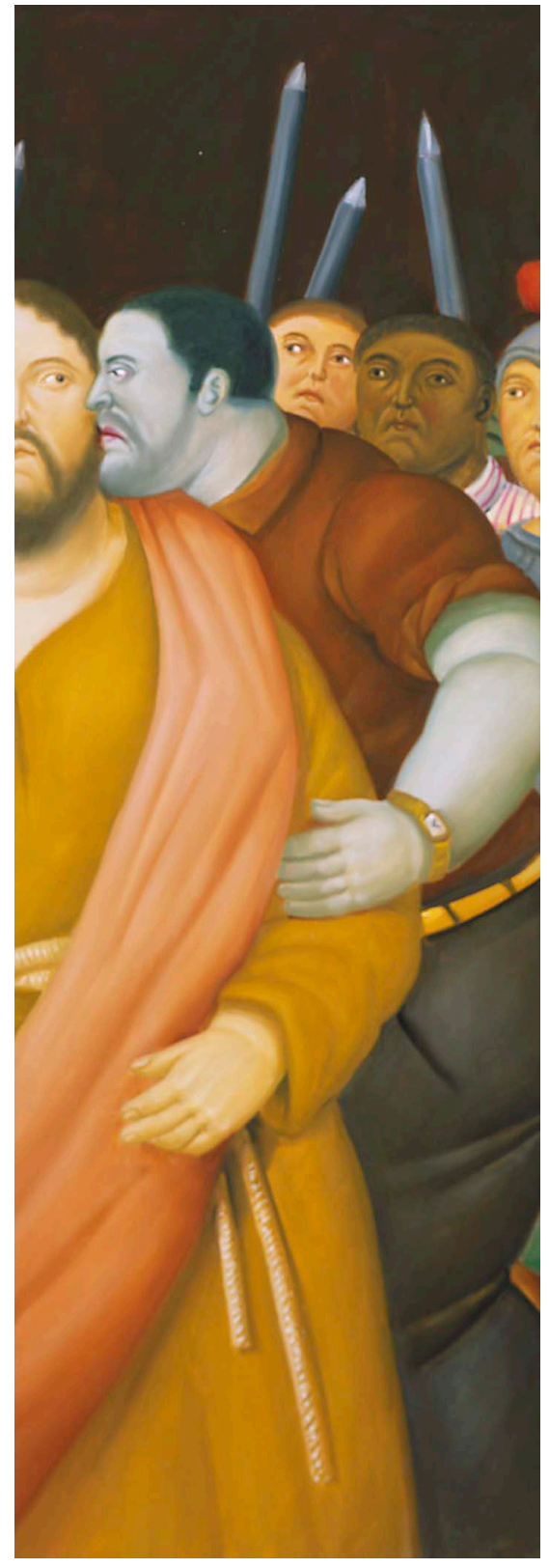

En el caso de la enseñanza, hay muchas interrogantes a las que hoy día urge dar respuesta para hacer de esta tarea una actividad con sentido, propósito y rumbo definido salir del estado de desarticulación y fragmentación del saber contemporáneo y de un pensamiento social y político, cuyos modos simplificadores han producido un efecto de sobra conocido y sufrido por la humanidad tanto en el presente como en el pasado. Por otro lado, es preciso que al operar procesos educativos se tomen en cuenta la problemática de la conciencia del inacabamiento en el obrar de la que nos habla Morín.

\section{EJE 2. INVESTIGACIÓN DOCENCIA}

Este segundo eje que se propone para innovar la tarea de la enseñanza está orientado a ver en la investigación la gran herramienta no sólo para la generación de nuevos conocimientos, sino también para transformar la práctica docente. De manera ordinaria todo proceso de investigación surge de las preguntas que un investigador se plantea y a las cuales desea dar una respuesta. En el caso de la enseñanza, hay muchas interrogantes a las que hoy día urge dar respuesta para hacer de esta tarea una actividad con sentido, propósito y rumbo definido, entre las cuales resaltan qué métodos son más eficaces para enseñar, qué actividades de aprendizaje pueden ser más significativas, cuáles técnicas dan mejores resultados en la interacción pedagógica, entre otras. En el caso del proyecto RECREA, este segundo eje tiene su fundamento en la conciencia de que en todas las instituciones universitarias se menciona que la docencia y la investigación son funciones sustantivas, dando como resultado una articulación de la mismas, siendo objeto de interés para los investigadores en el campo de la educación, aduciendo que este vínculo es positivo y necesario (Hernández- Piña, Tesouro \& Puiggali, 2015). 


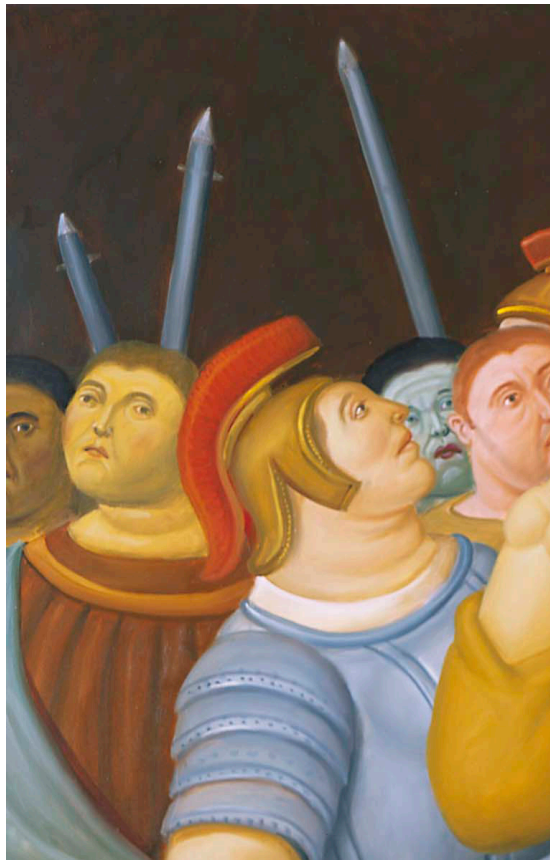

La relación entre docencia e investigación permite concebir a todo académico como aquel que no sólo conduce procesos instruccionales, sino además como un agente activo que investiga sobre su propia práctica, puesto que al paso del tiempo y su desarrollo como docente, reflexiona, indaga y teoriza sobre lo que hace como actividad profesional y los resultados que obtiene de ella.

Podemos afirmar entonces sin lugar a dudas que la relación que guarda la investigación con la docencia es asimétrica, puesto que cuando más fluye en este sentido, la docencia se beneficia en gran medida de la actividad investigativa, ya que de manera directa esta relación se fortalece cuando el profesor de manera directa comparte con sus estudiantes en el aula los avances y frutos que va recogiendo del proceso de investigación que lleva a cabo, los cuales están condensados en hechos, datos, reflexiones, etc., pero además, el beneficio es indirecto cuando en el profesor existe la aproximación al conocimiento, promueve

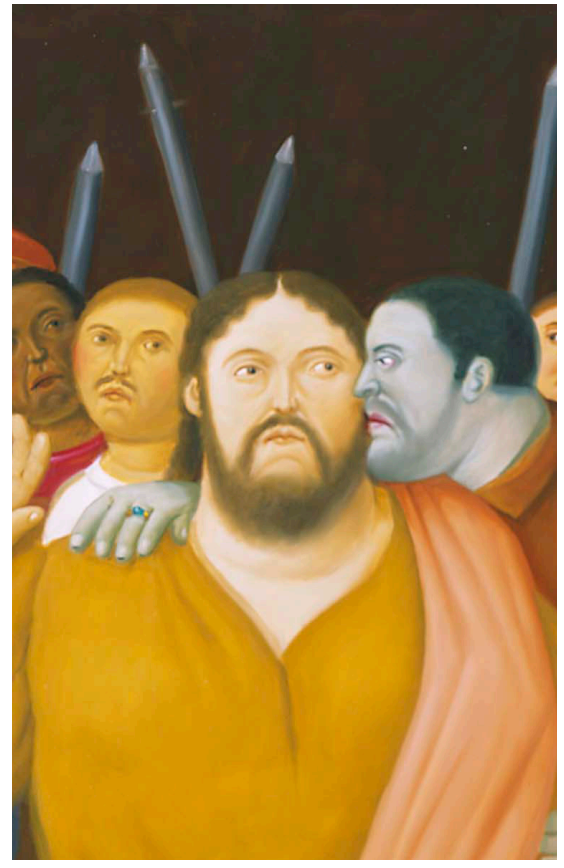

en sus alumnos la indagación como un recurso para su propio aprendizaje (Orler, 2012). Autores como Griffiths (2004) señalan que también puede haber un beneficio desde la docencia a la investigación cuando el profesor puede trasladar hacia su línea de investigación la retroalimentación que recibe de sus alumnos.

Tomando como referencia las ideas de Griffiths (2004), éste propone diferentes estilos de enseñanza de acuerdo con su interacción con la investigación, mismos que se enuncian a continuación:

- La que es dirigida o guiada por la investigación: los estudiantes en ella aprenden conforme a los intereses de investigación de los académicos y en donde una parte considerable de la enseñanza hace énfasis en la trasmisión de información.

- La aproximada mediante procesos de investigación: Los estudiantes aprenden acerca de los procesos de investigación; el curso enfatiza tanto los procesos

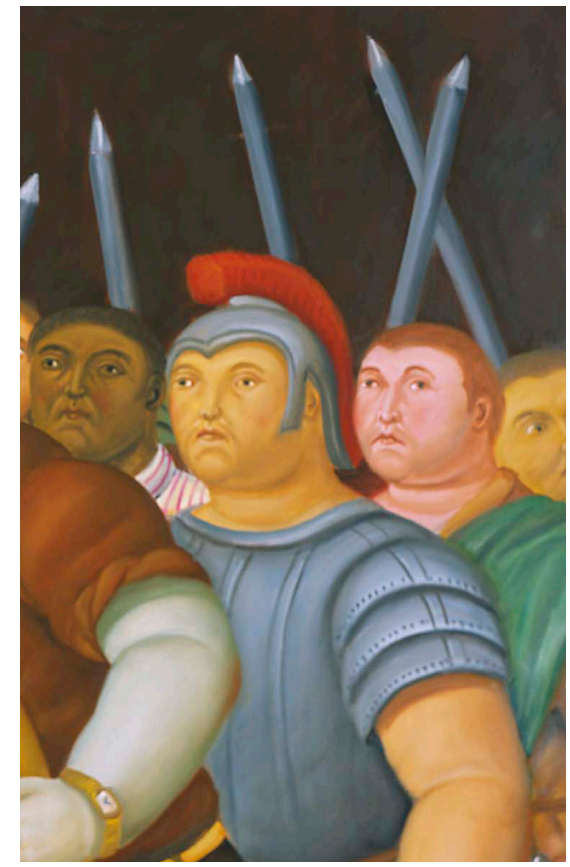

para la generación del conocimiento como el conocimiento que se ha logrado, y el profesor trata de generar una forma de comportamiento 0 actitud hacia la investigación a través de sus enseñanzas.

- Basada en la investigación: Los estudiantes aprenden investigando; el curso está diseñado en torno a actividades de indagación y búsqueda; el rol del profesor migra hacia un estatus de facilitador.

- Sustentada en la investigación del aprendizaje: implica la indagación sobre los propios procesos de enseñanza-aprendizaje donde está inmerso el profesor.

En virtud de lo anterior, para seguir fortaleciendo el vínculo investigación-docencia o fortalecerlo cada vez más, se requiere de una serie de acciones tanto institucionales como de los académicos, entre ellas el incentivar de igual forma las actividades docentes y de investigación, la profesionalización de los profesores en técnicas y métodos docentes y la formación de 
comunidades de aprendizaje de profesores que experimenten estrategias de vinculación investigación-docencia, incorporando en estas comunidades a expertos en investigación educativa.

Resumiendo este segundo eje podemos afirmar que la actividad académica incluye la realización de ambas actividades (docencia e investigación) y exige el fortalecimiento del vínculo entre ellas. Si bien es cierto que el trabajo del aula debe centrarse en el alumno, necesita seguir orientándose para fomentar la aproximación al conocimiento a través de la indagación, provocar los cuestionamientos y la búsqueda de soluciones a los problemas que aquejan el mundo de la enseñanza, y donde, sin duda alguna, la investigación es pieza clave.

\section{EJE 3.TECNOLOGÍAS \\ DE LA INFORMACIÓN}

Y COMUNICACIÓN EN LA EDUCACIÓN

A lo largo del último siglo y en el curso del presente, somos testigos de cómo las tecnologías digitales y multimedia se han incorporado en los diversos

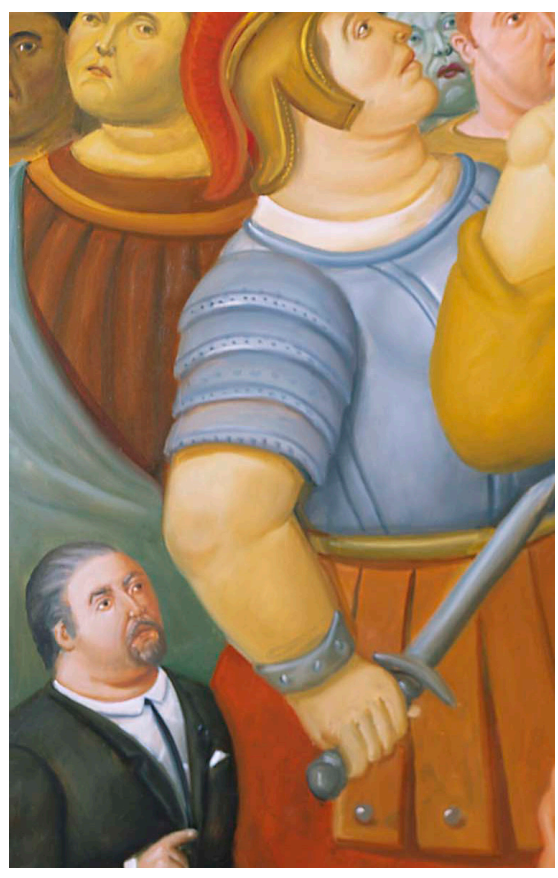

ámbitos de la vida personal, social, económica, educativa y en la generación de conocimiento, influenciando todos los ámbitos de la vida humana, permitiendo el desarrollo de habilidades en las personas, creando nuevas expectativas de vida y desarrollo para los jóvenes; más y mejores formas y oportunidades de trabajo; nuevas y variadas formas de interacción y comunicación, facilitando además los procesos de enseñanza y aprendizaje, haciéndolos más fluidos, ricos en contenido y significativos.

En este sentido, podemos vislumbrar que los desarrollos clave en la tecnología para la educación superior actual y para la que se irá desarrollando en los próximos años, será mediante las tecnologías adaptativas para el aprendizaje; el aprendizaje móvil, el internet de las cosas; la generación de LMS; la inteligencia artificial y los interfaces naturales para el usuario.

Actualmente podemos darnos cuenta de cómo los sistemas educativos cada vez más van incorporando la

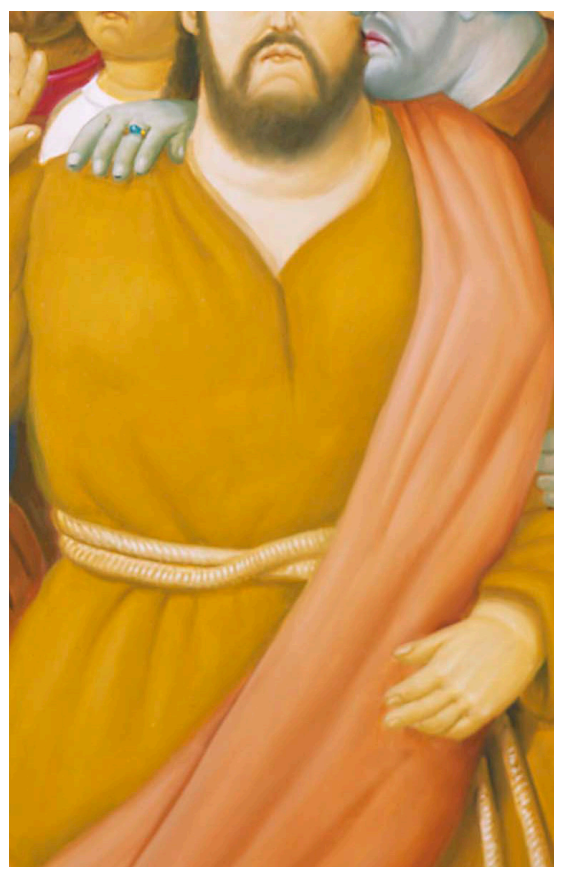

tecnología a las tareas no sólo de la enseñanza sino también del aprendizaje. Se desarrolla de manera gradual una cultura de digitalización que va permitiendo migrar de una educación netamente tradicional a una donde el mundo digital se impone de manera contundente.

Hay quienes incluso afirman que se deben llevar a cabo procesos de alfabetización digital que permitan a los académicos prepararse y estar en condiciones de educar a generaciones de jóvenes que ya nacieron con la tecnología, mientras que estos otros necesitan ir aprendiendo y apropiándose de ella.

Dentro de estos cambios y transformaciones que se dan en el contexto educativo, podemos apreciar que la virtualización es parte de la transformación digital que impulsa la revolución tecnológica, en la cual el derecho a la educación virtual se constituye como un derecho educativo fundamental. Cada vez más los países de América Latina van creando sistemas

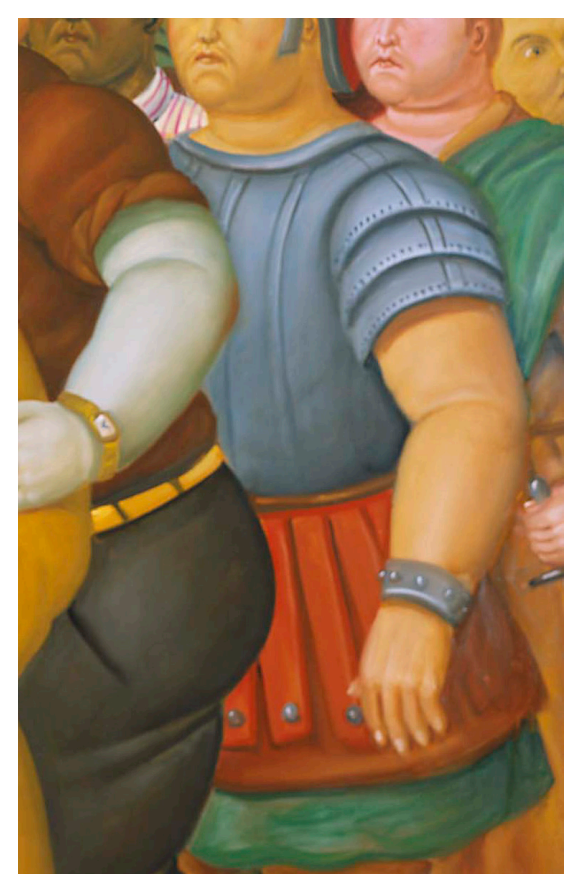


de educación a distancia para permitir el acceso a la educación de algunos determinados grupos y poblaciones.

Toda vez que se fue constituyendo de manera gradual y articulada el proyecto RECREA en cada una de las regiones de México que fueron determinadas para tal fin, la siguiente fase fue la aplicación de la metodología en los cursos que teníamos a su cargo los académicos y académicas involucrados en este proyecto, tanto en la Facultad de Filosofía y Letras de la UANL, en la Facultad de Salud Pública y Nutrición y en la Escuela Normal Miguel F. Martínez, todas ellas ubicadas en Monterrey, NL., y a quienes se nos dio un plazo de medio año para la aplicación de la misma y la obtención de resultados. Inicialmente los académicos participantes tuvimos la necesidad de reconstruir muchas de nuestras ideas pedagógicas, formas de entender el diseño instruccional, formas de operar y desarrollar nuestros cursos en el aula y la adecuación de nuestras formas de trabajo a las necesidades del contexto actual. Por otro lado, hubo la necesidad de capacitarse en temáticas como el pensamiento complejo, la investigación-acción dentro de nuestra propia práctica y ver la tecnología y recursos digitales disponibles, herramientas útiles para operar de una manera más exitosa nuestros procesos como enseñantes.

Después de tener medio año donde pudimos aplicar el diseño instruccional trabajado desde la metodología del proyecto RECREA, pudimos constatar la innovación que se vivió en nuestros cursos desde esta nueva mirada pedagógica en el diseño instruccional, adentrándonos cada vez más en una cultura de educación disruptiva don-

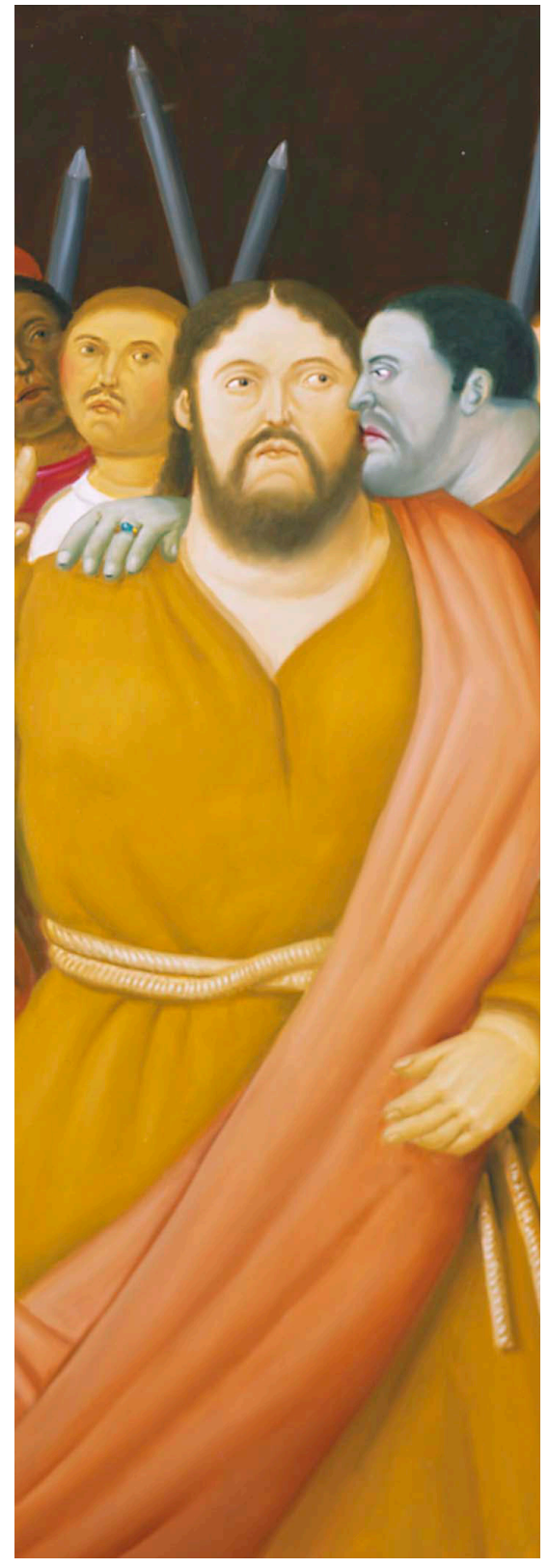

de, maestros y estudiantes, pudimos romper con formas tradicionales de hacer las cosas, arribar a otras concepciones de enseñanza y aprendizaje y darnos cuenta que siempre es posible hacer las cosas de manera diferentes.

Cabe destacar que el fruto de la aplicación de la metodología RECREA en las aulas, fue la conformación de las comunidades de práctica donde los académicos participantes en el proyecto hemos podido reflexionar $y$ compartir sobre nuestras experiencias de trabajo para enriquecernos con las experiencias de cada uno, vividas desde la práctica. Si bien es cierto que el trabajo de los académicos no deja de ser un trabajo individual, el proyecto RECREA promueve un acercamiento entre académicos donde, en un clima de respeto y reconocimiento al ser y quehacer del otro, se puede compartir la experiencia, logros y retos que se experimentan en el desarrollo cotidiano de la profesión.

Conviene señalar que a la fecha se sigue aplicando la metodología en los cursos que los académicos que pertenecemos a RECREA tenemos a nuestro cargo. Estamos en una fase de recupe-
Desde la metodología del proyecto RECREA, pudimos constatar la innovación que se vivió, arribar a otras concepciones de enseñanza y aprendizaje y darnos cuenta que siempre es posible hacer las cosas de manera diferentes 
ración de resultados que vamos obteniendo, ya que los trabajos de este proyecto siguen en marcha y se ofrecerán datos concretos y estadísticos de los frutos que se han ido alcanzado durante estos periodos de aplicación de la propuesta metodológica.

\section{CONCLUSIONES}

Podemos afirmar que uno de los resultados más significativos ha sido sin duda alguna el acercamiento entre académicos normalistas y académicos universitarios, donde hemos podido, desde la naturaleza e identidad de las instituciones a las que pertenecemos, compartir experiencias pedagógicas y enriquecernos con las áreas especializadas de formación trabajadas en nuestras instituciones.

RECREA, como proyecto y propuesta, ha generado como resultado una nueva cultura académica que de forma gradual se va compartiendo y desarrollando en las universidades y escuelas normales de México. Consideramos que de manera incipiente estos son unos de los primeros resultados a nivel de la comprensión, planeación y puesta en práctica de un nuevo diseño instruccional; sin duda alguna, habrá logros más significativos con el paso del tiempo.

Toda vez que hemos podido discurrir en la necesidad de la transformación de los sistemas educativos, no podemos dejar fuera que necesitamos impulsar un cambio de mentalidad en los agentes educativos para que puedan abrirse a las nuevas alternativas $y$ maneras de hacer educación, con una visión de futuro.

La cultura del cambio y la innovación necesita permear de manera gradual en las instituciones educativas con el fin de ajustarlas a las nuevas necesidades del entorno, haciendo que los procesos de enseñanza y aprendizaje que se viven al interior de las mismas sean significativos para los educandos.

Sin lugar a duda RECREA es una alternativa ideal para responder a la capacitación de los docentes de todos los niveles educativos, ayudándoles a repensar su práctica, innovar la planeación didáctica, hacer más eficientes sus intervenciones en el aula y repensar la enseñanza en pro de favorables procesos de aprendizaje más significativos en los docentes.

Cada uno de los ejes propuestos por RECREA favorecen los cambios tanto en la manera de enseñar como de aprender: cada uno de ellos propone nuevas formas de entender la acción educativa y los resultados ideales que habría que esperar de ella.

Si bien es cierto que los maestros y maestras somos expertos en algún área de conocimiento, finalmente tenemos la consigna de seguir aprendiendo buscando arribar a nuevos horizontes pedagógicos, y preparándonos para enfrentar un entorno que cada vez demanda de nosotros mayor especialización. Hemos querido dar a conocer por medio de esta ponencia la propuesta metodológica para estructurar, orientar y operar los procesos de enseñanza y aprendizaje de nuestros estudiantes para hacer más eficaz nuestra tarea en el aula.

Como proyecto, RECREA finalizará en el año 2021 y es allí donde de manera contundente se podrán conocer las bondades, frutos, éxitos 0 áreas de oportunidad de la aplicación de esta propuesta en los procesos de instrucción. No obstante, aun cuando estamos todavía en el arranque de las fases iniciales, creemos que se comienzan a notar los frutos de esta nueva forma de planear y hacer educación, considerando que la teoría de la complejidad, la investigación-acción llevada a cabo en nuestra práctica y el uso de las tecnologías de la información y comunicación, darán rumbo certero a nuestras funciones como agentes educativos.

Nada es permanente, excepto el cambio, así lo afirmó Heráclito, filósofo de la Grecia Antigua. Hoy es una consigna para nosotros los educadores y educadoras; necesitamos cambiar e innovar nuestras prácticas, sistemas, formas de ser y pensar la educación; es una tarea, una consigna, una forma de vida profesional.

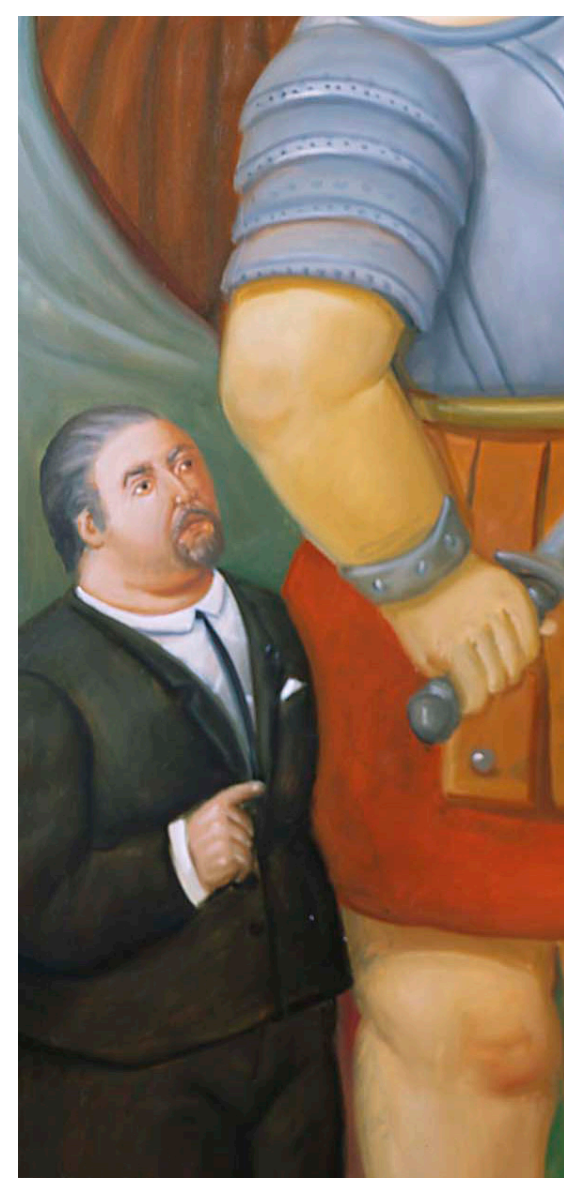




\section{REFERENCIAS}

Bausela, E. (2004). La docencia a través de la investigación-acción. Revista Iberoamericana de Educación. Recuperado de http://rieoei.org/profesion25.htm Gulikers, J.T.M., Bastiaens, T.J.

Elliot.J (1993). Cambio educativo desde la investigación acción. Morata: España. Golberg, E. (2018). El cerebro humano en la era de la innovación. España: Crítica. Morín, E. (2002). Educar en la era planetaria. Barcelona: Editorial Gedisa. Norton, Lin S. (2004). La epistemología de la complejidad. Gazeta de Antropología, 20. Recuperado de http://www. Ugr.es/pwla

Merrieboer, J.G.y Kischner, P.A. (2010). Diez pasos para el aprendizaje complejto: un acercamiento sistemática al diseño instruccional de los cuatro componentes. España: Publisher.

Padilla C. M. T., Gil F. J. (2008). La evaluación orientada al aprendizaje en la Educación Superior: condiciones y estrategias para su aplicación en la docencia universitaria. Revista española de pedagogía. Año LXVI, no. 241, sept-dic.

Hernández-Piña, Tesouro \& Puiggali, (2015). Los enfoques del aprendizaje, recuperado de: https://www.redalyc.org/pdf/2833/283331396013.pdf

Griffiths, Abrahams (2004). El estilo de la enseñanza en la cátedra universitaria, recuperado de: http://repositorio.filo.uba.ar/bitstream/handle/filodigital/6048/ uba_ffyl_t_2013_893624_v2.pd f?sequence=2\&isAllowed=y

Orler, José (2012). Docencia e investigación, recuperado de: http://www.derecho.uba.ar/publicaciones/rev_academia/revistas/19/docencia-investigacionuna-relacion-antagonica-inexistente-o-necesaria.pdf

Taeli Gómez, F, (2012). El nuevo paradigma de la complejidad y la educación: una mirada histórica. Polis [En ligne], 25 | 2010, Recuperado de http://polis. revues.org/400

Torres, Luis Carlos (2013). La complejidad humana, recuperado de: https://disi. unal.edu.co/ Ictorress/tgs/Tgs003.pdf

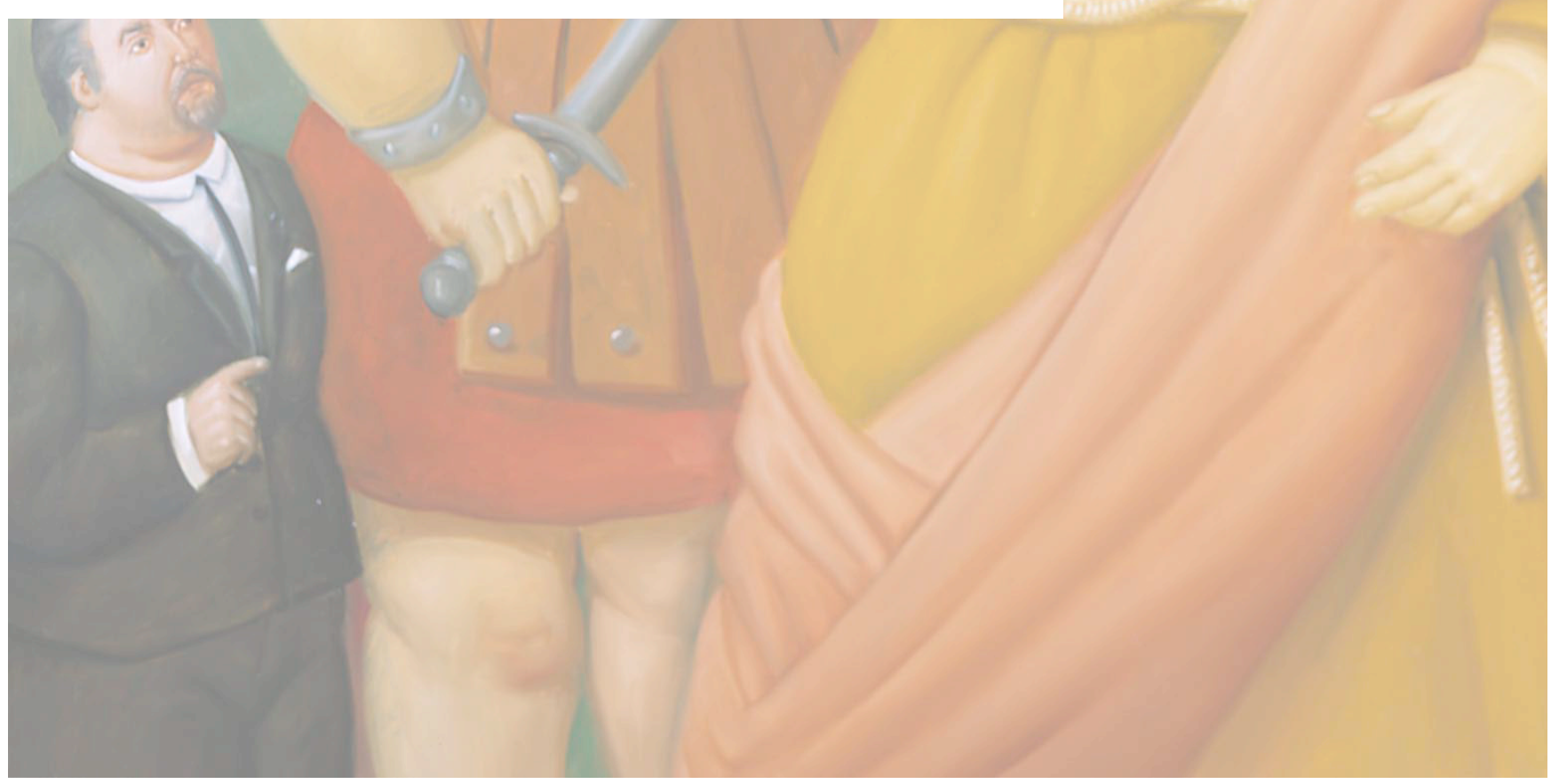




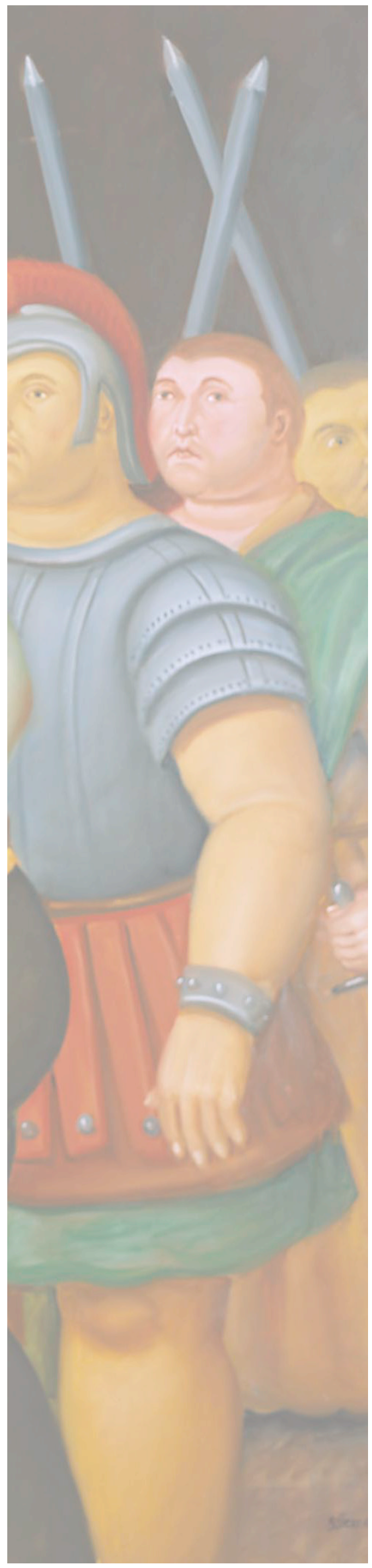

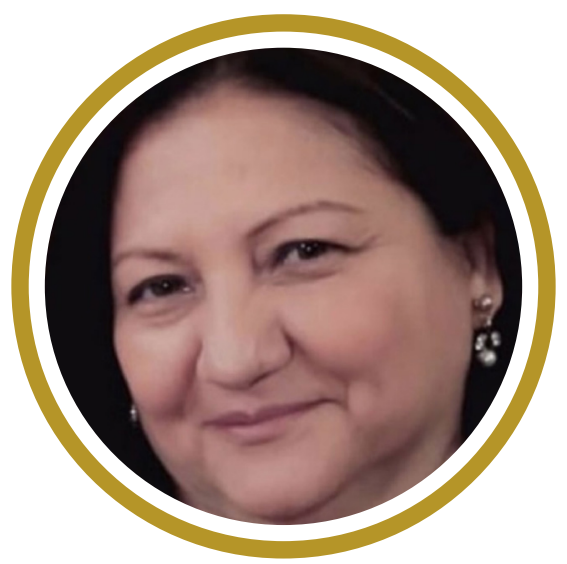

Emma Isabel Rodríguez Ruvalcaba

Licenciada en Pedagogía y con maestría en Enseñanza Superior, en la facultad de Filosofía y Letras de la Universidad Autónoma de Nuevo León. Se desempeña como Maestra en la misma facultad, en el Colegio de Educación en el área de Didáctica y Evaluación del Currículo y está encargada de la Secretaría de Planeación y Evaluación de la Facultad de Filosofía y Letras UANL.

\section{Correo Electrónico:}

docenteemma@hotmail.com

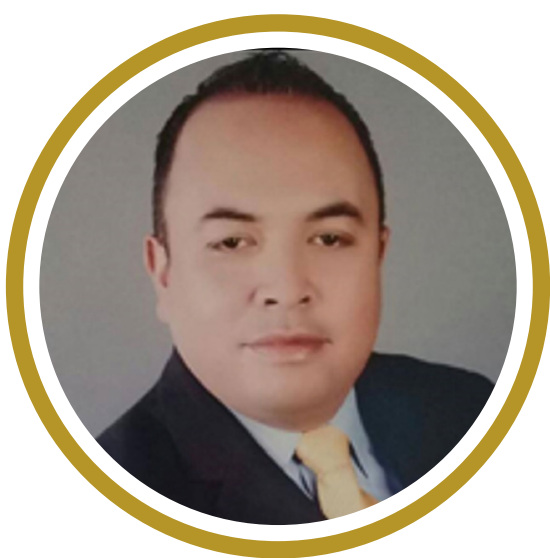

Felipe

Abundis de León

Licenciado en Pedagogía. Maestro en Ciencias con especialidad en Educación. Doctor en Filosofía con acentuación en estudios de la Educación. Miembro del Colegio de Pedagogos de México. Miembro de la Sociedad Española de Pedagogía. Miembro de la Red de Investigadores de la UANL. Miembro del Comité de Diseño y Creación de Nuevos Programas de Posgrado a Nivel Maestría. Coordinador Operativo para la región noreste del país del programa RECREA (Red de Comunidades para la Renovación de los Procesos de Enseñanza-Aprendizaje). Actualmente, coordinador del Colegio de Educación en la Facultad de Filosofía y Letras de la UANL.

\section{Correo Electrónico:}

felipe.abundisdln@uanl.edu.mx

Fecha de Recepción: 15/03/20

Fecha de Aprobación: 15/08/20 